\title{
Correlation of the anatomical sacral slope with pelvic incidence in female patients with developmental hip dysplasia: a retrospective cross-sectional study
}

\author{
Norio Imai ${ }^{1^{*}}$, Hayato Suzuki ${ }^{2}$, Atsushi Sakagami², Yuki Hirano ${ }^{1,2}$ and Naoto Endo ${ }^{1,2}$
}

\begin{abstract}
Background: The anatomical sacral slope is considered as an anatomical pelvic parameter independent of femoral head centers for measurement of anatomical sacral slope and was previously described to strongly correlate with pelvic incidence on a two-dimensional examination of healthy subjects. However, the correlation between anatomical sacral slope and pelvic incidence was unclear in patients with developmental dysplasia of the hip. This study aimed to examine the correlation between anatomical sacral slope and other spinopelvic parameters by analyzing plain radiographs of female patients with developmental dysplasia of the hip.

Methods: Eighty-four women with developmental dysplasia of the hip were examined. Lumbar lordosis, thoracic kyphosis, pelvic incidence, sacral slope, and anatomical sacral slope (the angle formed by the straight line of the S1 superior endplate and a line at a right angle to the anterior pelvic plane) were determined by analyzing plain radiographs. The correlations were examined by Pearson's correlation coefficients, and intra- and inter-rater intraclass correlation coefficients were evaluated for reliability.

Results: A strong correlation was observed between pelvic incidence and anatomical sacral slope $(r=0.725, p<$ 0.001). In addition, the correlation between anatomical sacral slope and lumbar lordosis was similar to that between pelvic incidence and lumbar lordosis ( $r=0.661, p<0.001$, and $r=0.554, p<0.001$, respectively). The intra-rater intraclass correlation coefficient values were 0.869 and 0.824 for anatomical sacral slope and pelvic incidence, respectively. Furthermore, the inter-rater intraclass correlation coefficient values were 0.83 and 0.685 for anatomical sacral slope and pelvic incidence, respectively.

Conclusions: We observed that the strong correlation between anatomical sacral slope and pelvic incidence in patients with developmental dysplasia of the hip was equal to that in normal healthy subjects. The correlation between anatomical sacral slope and lumbar lordosis was equal to that between pelvic incidence and lumbar Iordosis. Additionally, the intraclass correlation coefficient values for the anatomical sacral slope were slightly higher than those for pelvic incidence. Thus, we conclude that anatomical sacral slope can be considered as a helpful (Continued on next page)
\end{abstract}

\footnotetext{
* Correspondence: imainorio2001@yahoo.co.jp

'Division of Comprehensive Musculoskeletal Medicine, Niigata University Graduate School of Medical and Dental Sciences, 1-757, Asahimachi-dori, Chuo ku, Niigata City, Niigata Prefecture 951-8510, Japan

Full list of author information is available at the end of the article
} changes were made. The images or other third party material in this article are included in the article's Creative Commons licence, unless indicated otherwise in a credit line to the material. If material is not included in the article's Creative Commons licence and your intended use is not permitted by statutory regulation or exceeds the permitted use, you will need to obtain permission directly from the copyright holder. To view a copy of this licence, visit http://creativecommons.org/licenses/by/4.0/. The Creative Commons Public Domain Dedication waiver (http://creativecommons.org/publicdomain/zero/1.0/) applies to the data made available in this article, unless otherwise stated in a credit line to the data. 
(Continued from previous page)

anatomical pelvic parameter that is a substitute for pelvic incidence not only in normal healthy subjects, but also in patients with developmental dysplasia of the hip.

Keywords: Anatomical sacral slope, Developmental dysplasia of the hip, Lumbar lordosis, Pelvic incidence

\section{Background}

Based on previous studies, it was considered that pelvic morphology, as well as pelvic incidence (PI), influences sagittal spinal alignments, such as lumbar lordosis (LL), sacral slope (SS) [1-3], and standing posture [4-6]. A larger PI is considered to be a risk factor for spondylolisthesis because it seemingly leads to anterior deviation of the sagittal vertical axis [7, 8]. Additionally, the discrepancy between PI and LL leads to spinal deformity in adults [9]. In sagittal spinal malalignment, maintaining a suitable balance is considered difficult, and it may lead to "hip-spine syndrome" [10]. Consequently, PI is considered as one of the most important clinical parameters and should be evaluated.

Generally, many surgeons evaluate sagittal thoracolumbar spinal alignment and pelvic parameters by analyzing two-dimensional (2D) plain radiographs captured in the standing position $[11,12]$. SS and pelvic tilt (PT) are defined as functional parameters, since these angles are influenced by the anteroposterior tilt of the pelvis, that is, anterior or posterior tilt in the sagittal plane in the standing position. On the contrary, PI is deemed to be an anatomical parameter as it is not influenced by the anteroposterior tilt of the pelvis. SS and PT are related to $\mathrm{PI}$ in geometrical relation by the formula $\mathrm{PI}=\mathrm{SS}+$ PT.

We recently described a correlation between PI and anatomical sacral slope (a-SS), SS relative to anterior pelvic plane (APP), in normal healthy subjects using $2 \mathrm{D}$ and three-dimensional (3D) measurements $[1,13$, 14]. The a-SS was considered as an anatomical parameter that does not require femoral head measurements, as is the case when determining the PI. This is advantageous as the femoral head center is sometimes difficult to establish. Previously, we observed a close correlation between PI and a-SS among normal healthy subjects and patients with developmental dysplasia of the hip (DDH) measured using only the 3D method [13]. However, the correlation between PI and a-SS has not been examined in patients with $\mathrm{DDH}$, wherein pelvic and/or spinal morphological features observed using 2D measurements may be different from those of normal subjects.

This study aimed to examine the correlation between PI and a-SS using plain radiographs of patients with DDH. Similarly, we examined the correlation between aSS and LL using 2D measurements.

\section{Methods}

Eighty-four women with bilateral DDH, who had undergone curved periacetabular osteotomy [15] for treating early-stage hip osteoarthritis due to DDH from April 1, 2010, to July 30, 2017, were examined in our hospital. The inclusion criterion was a center-edge angle of the hip joints less than $20^{\circ}$, obtained from the anteroposterior view of the hips on plain radiographs. This is because these patients seemed to have a common morphological characteristic of DDH in their pelvis and might have a common functional alignment in the pelvis and spine. We excluded subjects who had undergone any hip joint surgery, those who were evaluated to have hip dysplasia of Crowe stages 2-4 [16] regarding subluxation, or those in which arthritic change was evaluated as Tonnis grades 2-3 [17] observed on plain radiographs of the hip.

The Ethical Review Board of our institution approved this study and waived the need for informed consent because of the retrospective cross-sectional design of the study.

\section{Measurements of pelvic and thoracolumbar parameters}

The pelvic parameters, such as PI, SS, and a-SS, and the thoracolumbar parameters, such as thoracic kyphosis (TK) and LL, were measured using thoracic and lumbar plain radiographs including the pelvis in the standing position. PI was established as the angle formed by the line at a right angle to the superior endplate of $\mathrm{S} 1$ at its middle point and the line connecting this point to the axis linking the bilateral femoral heads (Fig. 1) [11]. The SS was established as the angle formed by the straight line of the S1 superior endplate and a leveled line at a right angle to the gravitational force direction. PT was established as the angle formed by the straight line connecting the middle point of the S1 endplate to the hip axis and the vertical line parallel to the direction of gravitational force (Fig. 1). Further, LL was established as the angle from the line of T12 inferior endplate and the line of the S1 superior endplate (Fig. 2). TK was defined as the angle formed by the line of the T1 superior endplate and the line of the T12 inferior endplate (Fig. 2).

\section{Statistical analysis}

We analyzed the data using SPSS software (version 24; SPSS, Inc., Chicago, IL). The correlation of PI, SS, a-SS, 


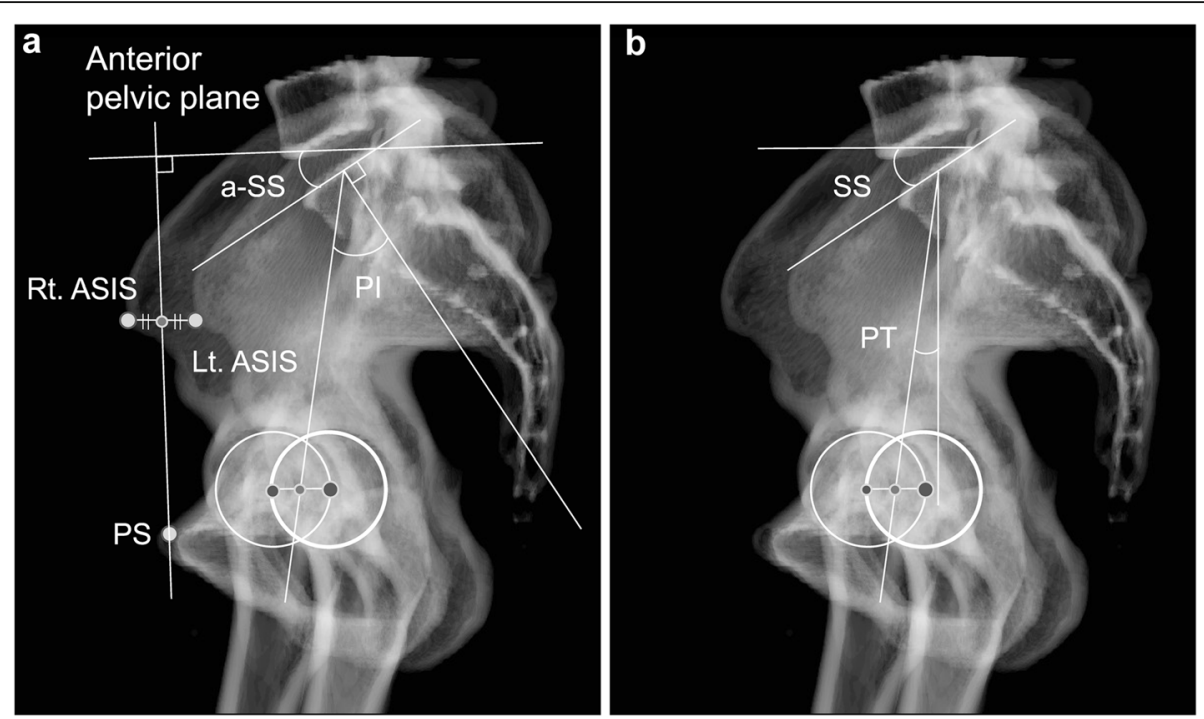

Fig. 1 Anatomical and functional parameters of the pelvis. a Anatomical parameters. b functional parameters. PI, pelvic incidence; SS, sacral slope; APP, anterior pelvic plane; L- and R-ASIS, left and right anterior superior iliac spine

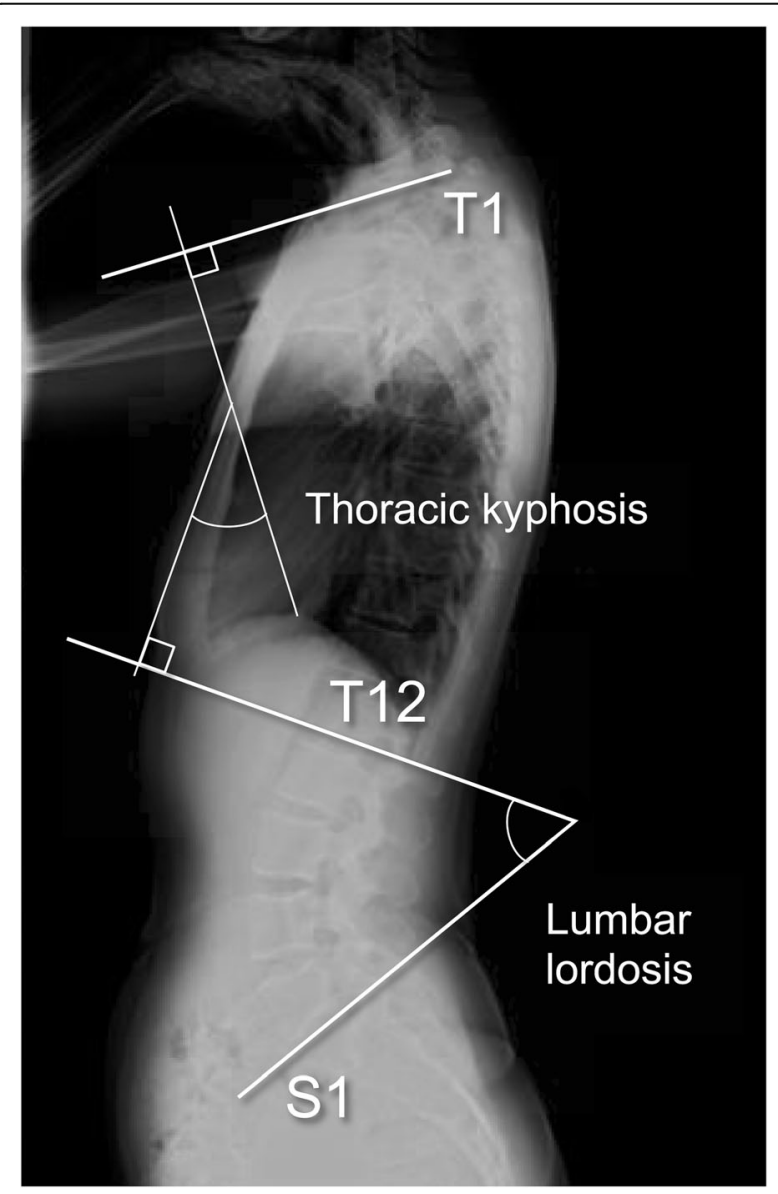

Fig. 2 Sagittal thoracolumbar spinal parameters. Lumbar lordosis (LL) was established by the T12 inferior end plate and the S1 superior end plate. Thoracic kyphosis (TK) is measured between the $\mathrm{T} 1$ superior end plate and the T12 inferior end plate
LL, and TK were evaluated with Pearson's correlation coefficients according to Guilford's definition [18]. Similarly, we evaluated the statistical power (type II ( $\beta$ ) error) using a post hoc analysis, with 0.3 as the effect size $(d)$ and 0.05 as type I $(\alpha)$ error, for the correlation analysis. We evaluated the validity of this study by calculating the mean absolute difference (MAD), the variability by the standard deviation (SD), and the intra- and inter-rater reliabilities with interclass correlation coefficients (ICCs) (with $95 \%$ confidence intervals) by two-tailed analysis. We measured 1-week intervals twice to determine the intra-rater reliability and drew a parallel between the measurements examined by two other observers to assess the inter-rater reliability. Values of $p$ below 0.05 were considered statistically significant.

\section{Results}

The average age and body mass index of the participants were $35.0 \pm 9.2$ years (range 20-52 years) and $22.0 \pm 2.9$ $\mathrm{kg} / \mathrm{m}^{2}$ (range $16.2-27.8 \mathrm{~kg} / \mathrm{m}^{2}$ ), respectively.

Table 1 shows the details of the parameters. A close correlation was observed between PI and a-SS $(r=$ 0.725, $p<0.001$ ) (Fig. 3), as defined by Guilford [18] (Table 2). The regression formula calculated from this correlation is as follows: $\mathrm{PI}=0.8 \times \mathrm{a}-\mathrm{SS}+21$.

Regarding the correlation between pelvic and thoracolumbar parameters, a strong correlation was observed between SS and LL $(r=0.827, p<0.001)$. Concerning the anatomical parameters, the correlation between a-SS and LL was equal to that between PI and LL ( $r=0.554, p<$ 0.001 , and $r=0.661, p<0.001$, respectively) (Table 2). However, no correlation was observed between TK and PI, SS, or a-SS. The power analysis of the correlation 
Table 1 The details of spinopelvic and spinal parameters of the 84 patients with developmental dysplasia of the hip

\begin{tabular}{ll}
\hline PI & $\mathbf{5 4 . 2} \pm \mathbf{1 0 . 6}\left(\mathbf{3 1 . 0 - 7 7 . 0 ^ { \circ } )}\right.$ \\
\hline SS & $38.5 \pm 10.8\left(10.0-69.0^{\circ}\right)$ \\
PT & $15.7 \pm 7.0\left(-8.0\right.$ to $\left.27.0^{\circ}\right)$ \\
a-SS & $40.8 \pm 9.4\left(20.0-61.0^{\circ}\right)$ \\
TK & $35.0 \pm 10.7\left(7.0-83^{\circ}\right)$ \\
LL & $55.4 \pm 18.4\left(3.0-83.0^{\circ}\right)$ \\
\hline
\end{tabular}

Mean \pm standard deviation (range)

PI Pelvic incidence, SS Sacral slope, PT Pelvic tilt, $a$-SS Anatomical sacral slope TK Thoracic kyphosis, LL Lumbar lordosis

showed a power value of 0.803 . Intra-rater MADs ranged from $2.6^{\circ}$ for SS to $3.7^{\circ}$ for PI, and the smallest ICC was 0.708 for TK (Table 3). As regards the MADs, intrarater MADs were slightly smaller than the inter-rater MADs (the largest MAD was $4.5^{\circ}$ for PI), and the smallest ICC was 0.685 for PI (Table 3).

\section{Discussion}

In this study, a strong correlation was observed between PI and a-SS; consequently, PI was considered feasible of being estimated from a-SS. Moreover, the correlation between a-SS and LL was similar to that between PI and LL $(r=0.661, p<0.001$, and $r=0.554, p<0.001$, respectively). These results were similar to those of a study in patients with $\mathrm{DDH}$ using 3D measurements [13] and results in normal healthy subjects, obtained by $2 \mathrm{D}$
Table 2 Pearson's correlation coefficients of pelvic and sagittal spinal parameters

\begin{tabular}{|c|c|c|c|c|c|}
\hline & SS & PT & a-SS & LL & TK \\
\hline PI & $0.632^{*}$ & $0.341^{*}$ & $0.725^{*}$ & $0.554^{*}$ & -0.017 \\
\hline SS & & -0.229 & $0.698^{*}$ & $0.827^{*}$ & 0.141 \\
\hline PT & & & 0.128 & 0.034 & -0.068 \\
\hline a-SS & & & & $0.661^{*}$ & 0.057 \\
\hline
\end{tabular}

measurements [1]. Consequently, the relationships between a-SS and LL and between PI and LL were similar among patients with DDH and normal subjects. The mean values in patients with $\mathrm{DDH}$ were as follows: PI, $54.2^{\circ}$; SS, $38.5^{\circ}$; and LL, $55.43^{\circ}$. In normal adults, the measured values of PI, SS, and LL have been described to range between 44.6 and $57.7^{\circ}, 32.5$ and $41.4^{\circ}$, and 48.2 and $57.2^{\circ}$, respectively $[19,20]$. Formerly, PI had been reported to be strongly correlated to SS and also to LL in normal women [8]. Our findings were similar to those of previous results [13]. Therefore, the results of our study can be considered valid.

PI-LL discrepancy (PI-LL $\geq 11^{\circ}$ ) has been described to likely lead to disability in patients with spinal deformities [9]. Following spinal fusion surgery for lumbar degenerative diseases, PI-LL discrepancy reportedly leads to residual symptoms, such as lumbago and other disabilities

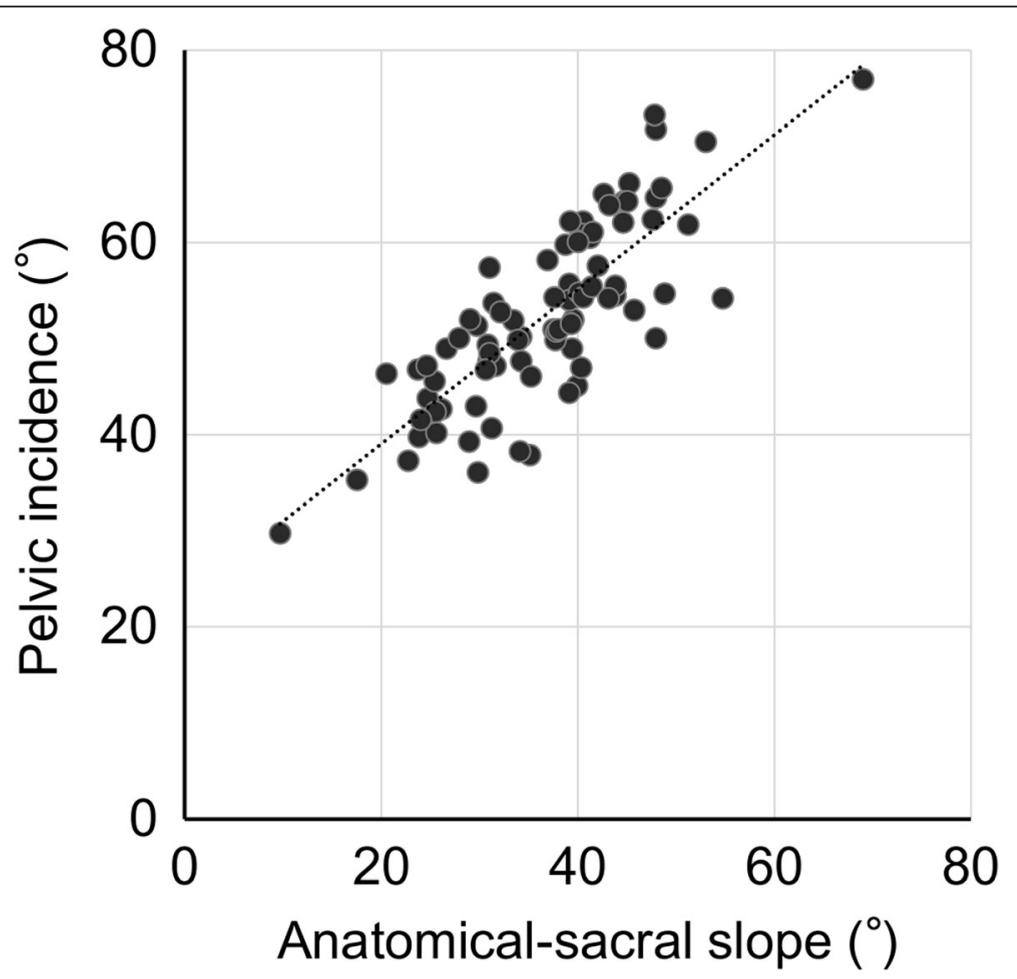

Fig. 3 The relationship between pelvic incidence and anatomical-sacral slope. Pelvic incidence was strongly related to anatomical-sacral slope 
Table 3 Intra- and interrater reliabilities of the measured values

\begin{tabular}{lll}
\hline & Intra-rater reliability & Inter-rater reliability \\
\hline $\mathrm{PI}$ & $3.7 \pm 2.8^{\circ}\left(0.824^{*}\right)$ & $4.5 \pm 3.6^{\circ}\left(0.685^{*}\right)$ \\
SS & $2.6 \pm 2.2^{\circ}\left(0.869^{*}\right)$ & $3.5 \pm 2.8^{\circ}\left(0.712^{*}\right)$ \\
PT & $2.9 \pm 2.8^{\circ}\left(0.842^{*}\right)$ & $4.0 \pm 3.2^{\circ}\left(0.697^{*}\right)$ \\
a-SS & $2.9 \pm 2.6^{\circ}\left(0.868^{*}\right)$ & $3.7 \pm 2.7^{\circ}\left(0.835^{*}\right)$ \\
TK & $3.4 \pm 3.0^{\circ}\left(0.708^{*}\right)$ & $3.9 \pm 3.8^{\circ}\left(0.698^{*}\right)$ \\
LL & $3.0 \pm 2.4^{\circ}\left(0.823^{*}\right)$ & $3.6 \pm 3.8^{\circ}\left(0.714^{*}\right)$
\end{tabular}

Mean absolute difference \pm standard deviation (intraclass correlation coefficient)

PI Pelvic incidence, SS Sacral slope, PT Pelvic tilt, $a$-SS Anatomical sacral slope, TK Thoracic kyphosis, LL Lumbar lordosis

${ }^{*} p<0.05$

$[21,22]$. Therefore, measuring the exact PI is essential. However, measuring PI requires identifying femoral head centers, which is sometimes arduous, especially in patients with aspherical femoral heads and with subluxation following osteoarthritis of the hip. In these patients, a new parameter, independent of the femoral head, seems to be required. Moreover, placing the acetabular component within a moderate level might be required to avoid increasing PI after total hip arthroplasty.

In this study, we observed that PI was strongly correlated to a-SS on $2 \mathrm{D}$ radiologic measurements. These findings were similar to those of several previous studies that described a strong correlation between PI and a-SS on 3D measurements in patients with DDH [13]. From these results, PI could be estimated from a-SS using the following regression formula: $\mathrm{PI}=0.8 \times \mathrm{a}-\mathrm{SS}+21$. Moreover, this formula is similar to that used in normal healthy subjects: $\mathrm{PI}=0.8 \times$ a-SS +18 [1]

Additionally, the findings showing a significant correlation between a-SS and LL were similar to those for PI and LL in patients with DDH and normal healthy subjects [13]. Therefore, a-SS may be useful to estimate PI in normal healthy subjects and patients with DDH and may be considered as a new anatomical pelvic parameter that is independent of the femoral head center for measurements. This is because measurements involving the femoral head were occasionally unsuitable, such as in patients with aspherical, flattening, or dislocated femoral heads.

Additionally, intra-rater and inter-rater MADs of PI, which included the femoral head center for measurements, were larger than those of a-SS, equal to those in normal subjects [13]. Therefore, a-SS may lead to higher reliability than PI when using $2 \mathrm{D}$ radiological measurements.

This study had several limitations. First, the sample size was small; however, the power value in the correlation analysis was 0.803 ; therefore, the sample size of this study was considered sufficient by power analysis. Second, this study only included Japanese individuals as participants. Previously, differences in sagittal thoracolumbar spinal and pelvic parameters among races had been described [23]. Therefore, our findings may be different from those in other races. Further studies are needed to investigate the generalizability of this result to other populations. Third, only female patients were included. DDH is predominant in females, with a female to male ratio of 9:1 [24]. Furthermore, < 20 male patients have undergone periacetabular osteotomy during the last 10 years in our hospital. Finally, we exclusively evaluated Crowe type 1 hip dysplasia; however, since the number of patients with high dislocation was less common, the influence seems not to be significant.

\section{Conclusions}

Anatomical sacral slope (a-SS), a novel parameter, can be considered convenient and can be examined by $2 \mathrm{D}$ plain radiographs. Additionally, the correlation between a-SS and LL was similar to that between PI and LL, and the $2 \mathrm{D}$ and $3 \mathrm{D}$ findings were similar in patients with DDH [13]. Thus, we believe that a-SS, which does not require the femoral head center for measurement, is a useful and a new suggested anatomical pelvic parameter that may be available instead of PI. Further large-scale studies are required to evaluate the validity and usefulness of a-SS as an anatomical parameter.

\section{Abbreviations \\ 2D: Two-dimensional; 3D: Three-dimensional; APP: Anterior pelvic plane; a- SS: Anatomical sacral slope; DDH: Developmental dysplasia of the hip; ICCs: Interclass correlation coefficients; LL: Lumbar lordosis; MAD: Mean absolute difference; PI: Pelvic incidence; PT: Pelvic tilt; SS: Sacral slope; TK: Thoracic kyphosis}

\section{Acknowledgements}

We would like to thank Editage (www.editage.com) for English language editing and publication support.

\section{Authors' contributions}

Conceptualization and formulation were done by $\mathrm{NI}, \mathrm{HS}, \mathrm{AS}, \mathrm{YH}$, and NE. Investigation and data collection were done by all the authors. $\mathrm{NI}$ carried out the statistical analysis. The study was performed under supervision of HS and NE. The authors read and approved the final manuscript.

\section{Authors' information}

Norio Imai, PhD, Professor of Division of Comprehensive Musculoskeletal Medicine, Niigata University Graduate School of Medical and Dental Sciences, Japan. Hayato Suzuki, PhD, Assistant professor of Division of Orthopedic Surgery, Department of Regenerative and Transplant Medicine, Niigata University Graduate School of Medical and Dental Sciences, Japan.

\section{Funding}

Not applicable.

\section{Availability of data and materials}

All data generated or analyzed during this study are included in this published article.

\section{Ethics approval and consent to participate}

The ethical review board of our institution approved this study (No. 20170344) and waived the need for informed consent because of the retrospective, cross-sectional design of the study. 


\section{Consent for publication}

Not applicable.

\section{Competing interests}

The authors declare that they have no competing interests.

\section{Author details}

'Division of Comprehensive Musculoskeletal Medicine, Niigata University Graduate School of Medical and Dental Sciences, 1-757, Asahimachi-dori, Chuo ku, Niigata City, Niigata Prefecture 951-8510, Japan. ${ }^{2}$ Division of Orthopedic Surgery, Department of Regenerative and Transplant Medicine, Niigata University Graduate School of Medical and Dental Sciences, 1-757, Asahimachi-dori, Chuo ku, Niigata City, Niigata Prefecture 951-8510, Japan.

Received: 5 June 2020 Accepted: 14 October 2020

Published online: 21 October 2020

\section{References}

1. Suzuki H, Imai N, Nozaki A, Hirano Y, Endo N. Anatomical sacral slope, a new pelvic parameter, is associated with lumbar lordosis and pelvic incidence in healthy Japanese women: a retrospective cross-sectional study. J Orthop Surg. 2020;28:1-5.

2. Vaz G, Roussouly P, Berthonnaud E, Dimnet J. Sagittal morphology and equilibrium of pelvis and spine. Eur Spine J. 2002;11:80-7.

3. Roussouly P, Gollogly S, Berthonnaud E, Dimnet J. Classification of the normal variation in the sagittal alignment of the human lumbar spine and pelvis in the standing position. Spine (Phila Pa 1976). 2005;30:346-53.

4. Imai N, Suzuki H, Nozaki A, Miyasaka D, Tsuchiya K, Ito T, et al. Evaluation of anatomical pelvic parameters between normal, healthy men and women using three-dimensional computed tomography: a cross-sectional study of sex-specific and age-specific differences. J Orthop Surg Res. 2019;14:126.

5. Schwab F, Patel A, Ungar B, Farcy JP, Lafage V. Adult spinal deformitypostoperative standing imbalance: how much can you tolerate? An overview of key parameters in assessing alignment and planning corrective surgery. Spine. 2010;35:2224-31.

6. Roussouly P, Nnadi C. Sagittal plane deformity: an overview of interpretation and management. Eur Spine J. 2010;19:1824-36.

7. Labelle H, Roussouly P, Berthonnaud E, Transfeldt E, O'Brien M, Chopin D, et al. Spondylolisthesis, pelvic incidence, and spinopelvic balance: a correlation study. Spine (Phila Pa 1976). 2004;29:2049-54.

8. Lafage V, Schwab F, Skalli W, Hawkinson N, Gagey PM, Ondra S, et al. Standing balance and sagittal plane spinal deformity: analysis of spinopelvic and gravity line parameters. Spine (Phila Pa 1976). 2008;33:1572-8.

9. Schwab FJ, Blondel B, Bess S, Hostin R, Shaffrey Cl, Smith JS, et al. Radiographical spinopelvic parameters and disability in the setting of adult spinal deformity: a prospective multicenter analysis. Spine (Phila Pa 1976). 2013;38:E803-12.

10. Offierski CM, MacNab I. Hip-spine syndrome. Spine. 1983:8:316-21.

11. Legaye J, Duval-Beaupère G, Hecquet J, Marty C. Pelvic incidence: a fundamental pelvic parameter for three-dimensional regulation of spinal sagittal curves. Eur Spine J. 1998;7:99-103.

12. Marty C, Boisaubert B, Descamps H, Montigny JP, Hecquet J, Legaye J, et al. The sagittal anatomy of the sacrum among young adults, infants, and spondylolisthesis patients. Eur Spine J. 2002;11:119-25.

13. Imai N, Miyasaka D, Tsuchiya K, Suzuki H, Ito T, Minato I, et al. Evaluation of pelvic morphology in female patients with developmental dysplasia of the hip using three-dimensional computed tomography: a cross-sectional study. J Orthop Sci. 2018;23:788-92.

14. Imai N, Ito T, Suda K, Miyasaka D, Endo N. Pelvic flexion measurement from lateral projection radiographs is clinically reliable. Clin Orthop Relat Res. 2013;471:1271-6.

15. Naito M, Nakamura Y. Curved periacetabular osteotomy for treatment of dysplastic hip. Clin Orthop Surg. 2014;6:127-37.

16. Crowe JF, Mani VJ, Ranawat CS. Total hip replacement in congenital dislocation and dysplasia of the hip. J Bone Joint Surg Am. 1979;61-A:15-23.

17. Tonnis D, Heinecke A. Acetabular and femoral anteversion: relationship with osteoarthritis of the hip. J Bone Joint Surg Am. 1999;81-A:1747-70.

18. Guilford JP, Fruchter B. editors. Correlation. Fundamental statistics in psychology and education. 5th ed. New York: McGraw-Hill; 1973.
19. Johnson RD, Valore A, Villaminar A, Comisso M, Balsano M. Sagittal balance and pelvic parameters-a paradigm shift in spinal surgery. J Clin Neurosci. 2013;20:191-6.

20. Zhu Z, Xu L, Zhu F, Jiang L, Wang Z, Liu Z, et al. Sagittal alignment of spine and pelvis in asymptomatic adults: norms in Chinese populations. Spine (Phila Pa 1976). 2014;39:E1-6.

21. Schwab F, Patel A, Ungar B, Farcy JP, Lafage V. Adult spinal deformitypostoperative standing imbalance: how much can you tolerate? An overview of key parameters in assessing alignment and planning corrective surgery. Spine (Phila Pa 1976). 2010;35:2224-31.

22. Aoki Y, Nakajima A, Takahashi H, Sonobe M, Terajima F, Saito M, et al. Influence of pelvic incidence-lumbar lordosis mismatch on surgical outcome of short-segment transforaminal lumbar interbody fusion. BMC Musculoskelet Disord. 2015;16:213.

23. Arima H, Dimar JR 2nd, Glassman SD, Yamato Y, Matsuyama Y, Mac-Thiong $J M$, et al. Differences in lumbar and pelvic parameters among African American, Caucasian and Asian populations. Eur Spine J. 2018;27:2990-8.

24. Agarwal A, Gupta N. Risk factor and diagnosis of developmental dysplasia of hip in children. J Clin Orthop Trauma. 2012;3:10-4.

\section{Publisher's Note}

Springer Nature remains neutral with regard to jurisdictional claims in published maps and institutional affiliations.

\section{Ready to submit your research? Choose BMC and benefit from:}

- fast, convenient online submission

- thorough peer review by experienced researchers in your field

- rapid publication on acceptance

- support for research data, including large and complex data types

- gold Open Access which fosters wider collaboration and increased citations

- maximum visibility for your research: over $100 \mathrm{M}$ website views per year

At BMC, research is always in progress.

Learn more biomedcentral.com/submissions 